\title{
Venus atmosphere profile from a maximum entropy principle
}

\author{
L. N. Epele ${ }^{1}$, H. Fanchiotti ${ }^{1}$, C. A. García Canal ${ }^{1}$, A. F. Pacheco ${ }^{2}$, and J. Sañudo ${ }^{3}$ \\ ${ }^{1}$ Laboratorio de Física Teórica, Departamento de Física, IFLP, Facultad de Ciencias Exactas, Universidad Nacional de La \\ Plata C.C. 67, 1900 La Plata, Argentina \\ ${ }^{2}$ Facultad de Ciencias and BIFI, Universidad de Zaragoza, 50009 Zaragoza, Spain \\ ${ }^{3}$ Departamento de Física, Universidad de Extremadura, Badajoz, Spain
}

Received: 25 April 2007 - Accepted: 6 July 2007 - Published: 18 October 2007

\begin{abstract}
The variational method with constraints recently developed by Verkley and Gerkema to describe maximumentropy atmospheric profiles is generalized to ideal gases but with temperature-dependent specific heats. In so doing, an extended and non standard potential temperature is introduced that is well suited for tackling the problem under consideration. This new formalism is successfully applied to the atmosphere of Venus. Three well defined regions emerge in this atmosphere up to a height of $100 \mathrm{~km}$ from the surface: the lowest one up to about $35 \mathrm{~km}$ is adiabatic, a transition layer located at the height of the cloud deck and finally a third region which is practically isothermal.
\end{abstract}

\section{Introduction}

Since the late 1950s, there have been hundreds of space exploration missions that have provided data on the composition, structure and circulation of planetary atmospheres. In particular, the vertical pressure-temperature profile of the atmosphere of Venus was accurately observed in the PioneerVenus II mission in 1978 (Seiff et al., 1980). In Landis et al. (2002), an averaged data of temperature, pressure and density of the Venus atmosphere as a function of altitude above the surface is given. In this paper we will use this information.

On Venus, the incoming solar energy, of the order of $2600 \mathrm{~W} / \mathrm{m}^{2}$, is almost twice as great as on Earth. Venus possess an atmosphere, which is about a hundred times as massive as that of the Earth and its surface temperature reaches $730 \mathrm{~K}$. Carbon dioxide is the major component (96.5 per cent), and 3.5 per cent of $\mathrm{N}_{2}$ is the next most abundant species.

In a recent paper by Verkley and Gerkema (2004), henceforth denoted by VG, have proposed a first-principles varia-

Correspondence to: L. N. Epele

(epele@ fisica.unlp.edu.ar) tional method to generate a $p-T$ profile for an atmospheric column. This profile is the result of maximizing the entropy of the column, $S$, maintaining fixed its mass, $M$, enthalpy, $H$, and the integral of the potential temperature, $L$. This method is flexible enough to describe from an isothermal profile to an adiabatic one depending on the value of a unique parameter. VG applied these ideas to the Earth's troposphere and accurately reproduced the U.S. Standard Atmosphere.

In their analysis, VG considered that the atmospheric gas is ideal, i.e. a gas that verifies the corresponding equation of state, and besides, the specific heats, $C_{V, p}$, are constant. When this second assumption is also fulfilled it is said that the gas is perfect. These two hypotheses are quite reasonable when dealing with the atmosphere of the Earth. For representative temperatures and pressures of the low Venus atmosphere, however, the temperature dependence of the $C_{V, p}$ has to inescapably be taken into account. As our goal in this paper is the application of the VG ideas to the Venus atmosphere, we have had to extend their original variational method to take into account this dependence of the specific heats on temperature. Consequently we will first extend the VG method to ideal imperfect gases. As is well known, the temperature dependence of the $C_{V, p}$ comes from the excitation of some internal vibrational levels of the molecule when the temperature is high enough.

Throughout the paper we will consider that $\mathrm{CO}_{2}$ is the main chemical component of the Venus atmosphere. The chemical impurities coming from the $\mathrm{N}_{2}$ produce negligible corrections in our analysis. Besides, we will not consider any correction coming from the interaction between the molecules, that is, we will stick to the scenario of an ideal gas but with the afore-mentioned temperature dependence of the $C_{V, p}$. Corrections of real gas in the Venus atmosphere have been considered (Staley, 1970) but for our purposes here they are negligible. Moreover, we will consider a constant gravity for the entire column under study because its variation with altitude has no impact in the results. The verification that the

Published by Copernicus Publications on behalf of the European Geosciences Union and the American Geophysical Union. 
equation of state of ideal gases is good to describe the gas of Venus can be done using the data of Landis et al. (2002). There one can check, at each height, that using pressure and temperature as input, the value of density obtained from the equation of the ideal gases is, in all cases, equal to the experimental value with a discrepancy lower than 2 per cent.

The index of the paper is as follows. In Sect. 2, we will recall the meaning of the potential temperature, $\theta$, for ideal perfect gases and its relation to the entropy. In Sect. 3, we will review the VG method and comment on the main results when it is applied to the Earth's troposphere. In Sect. 4, we will put in evidence the necessity of considering an explicit dependence of the $C_{V, p}$ with temperature for the $\mathrm{CO}_{2}$ molecule, to properly describe the Venus atmosphere. In Sect. 5, we will calculate the equation of the potential temperature, i.e. the form of an adiabatic, when dealing with ideal-imperfect gases. In Sect. 6, we will extend the VG method to ideal-imperfect gases. In Sect. 7, this new method will be applied to the Venus atmosphere and the results will be commented on. Finally, in Sect. 8 we present our conclusions.

\section{The potential temperature and the entropy for ideal perfect gases}

The first principle of Thermodynamics says that

$\delta U=\delta Q-p \delta V$.

Throughout this paper, $\delta$ indicates all the variations of thermodynamical quantities, whereas $d$ will be used to refer to variations of quantities at different altitudes.

For ideal gases, the equation of state, for $1 \mathrm{~mol}$, is

$p V=R^{*} T$

(where $R^{*}=8.3143 \mathrm{~J} \mathrm{~mol}^{-1} \mathrm{~K}^{-1}$ is the universal gas constant) and the specific internal energy (internal energy per unit mass, noted with lower case letter) reads

$u=C_{V} T+$ constant

In an adiabatic process, $\delta Q=0$, using the first two equations and remembering that $C_{p}=C_{V}+R^{*}$, we find

$C_{p} \frac{\delta T}{T}=R^{*} \frac{\delta p}{p}$.

Thus, two points $(p, T)$ and $\left(p_{r}, T_{r}\right)$ belonging to the same adiabatic trajectory fulfil

$C_{p} \int_{T_{r}}^{T} \frac{\delta T^{\prime}}{T^{\prime}}=R^{*} \int_{p_{r}}^{p} \frac{\delta p^{\prime}}{p^{\prime}}$.

In this case, the reference temperature $T_{r}$ becomes the so called potential temperature $\theta$ corresponding to the reference pressure $p_{r}$. Note that in the previous equations, $C_{p}$ has been considered as a temperature independent constant because in this section we are dealing with an ideal-perfect gas. This last equation constitutes one of the forms of expressing Poisson equation for an adiabatic process. Explicitly it reads as

$T p^{-\kappa}=T_{r} p_{r}^{-\kappa} ; \kappa=R^{*} / C_{p}$.

As it is standard, choosing the reference pressure $p_{r}=1000 \mathrm{mb}$, the potential temperature $\theta$ reads

$\theta=T\left(\frac{1000 \mathrm{mb}}{p}\right)^{\kappa}$.

The potential temperature of a gas is therefore the temperature that it would take if we compress or expand it adiabatically to a reference pressure of $1000 \mathrm{mb}$ (Tsonis, 2002). Obviously, the choice $p_{r}=1000 \mathrm{mb}$ is only a question of convenience when we are dealing with the low atmosphere of the Earth. In other words, the value of the reference pressure $p_{r}$ is arbitrary.

The differential expression for the specific entropy of ideal gases is

$\delta s=C_{p} \frac{\delta T}{T}-R^{*} \frac{\delta p}{p}$.

Because of the definition of $\theta$ in Eq. (7), $\delta \ln \theta=\delta \ln T-\kappa \delta \ln p$ and consequently

$\delta s=C_{p} \delta \ln \theta$,

and because we are considering that $C_{p}$ is constant

$s=C_{p} \ln \theta+$ constant.

Due to this direct relation, the potential temperature $\theta$ is usually considered as the entropy of the gas in the physics of the atmosphere. It is apparent that in a process of constant $\theta$, the entropy is also constant.

\section{The Verkley-Gerkema method}

VG have proposed a first-principles variational method to generate a $p-T$ profile for an atmospheric layer. In this method, one considers a column of dry air with unit horizontal area in hydrostatic equilibrium, bounded by two fixed values of the pressure. At each altitude $z$, the local thermodynamics is related to $p(z), T(z)$ and $\rho(z)$ that can be inverted into $z=z(p), T(p)$ and $\rho(p)$, since $d p / d z=-\mathrm{g} \rho$. The profile one is looking for comes out after the maximization of the entropy with the condition that the enthalpy and the vertical integral of the potential temperature, $L$, are kept fixed. As the top and bottom pressures, $p_{2}$ and $p_{1}$, of the column are 
specified, the total mass of air is also fixed. We denote by

$$
\begin{aligned}
S & =\frac{C_{p}}{g} \int_{p_{2}}^{p_{1}} \ln \theta d p \\
M & =\frac{1}{g} \int_{p_{2}}^{p_{1}} d p=\frac{1}{g}\left(p_{1}-p_{2}\right) \\
H & =\frac{C_{p}}{g} \int_{p_{2}}^{p_{1}} T d p \\
L & =\frac{C_{p}}{g} \int_{p_{2}}^{p_{1}} \theta d p
\end{aligned}
$$

the total entropy, mass, enthalpy, and vertical integral of the potential temperature (Bohren and Albrecht, 1998) of the air column, respectively. The requirement of $L$ fixed is appropriate in the case of convective mixing. An analysis on this issue and its bibliographic antecedents are presented in Verkley and Gerkema (2004).

As said above, VG imposed the maximization of $S$, with $M, H$, and $L$ fixed, namely

$\delta S+\lambda \delta H+\mu \delta L=0$,

$\lambda$ and $\mu$ being Lagrange multipliers. This means that one should maximizes the entropy with respect to the temperature profile of the atmosphere with the constraints imposed. The profile variation is consequently performed along the column through the variation of the temperature keeping the pressure fixed at every altitude.

From the maximization with constraints, VG deduced

$\frac{1}{T}+\lambda+\mu\left(\frac{p_{0}}{p}\right)^{\kappa}=0$.

Introducing

$\alpha=\frac{\mu}{\lambda}$,

and denoting the temperature at $p_{0}$ by $T_{0}$, the resulting $p-T$ profile is

$T=T_{0} \frac{1+\alpha}{1+\alpha\left(p_{0} / p\right)^{\kappa}}$.

This result is actually powerful; note that it is able to describe a wide range of profiles, from an isothermal profile, in the $\alpha \rightarrow 0$ limit, to an adiabatic profile in the limit of $\alpha \rightarrow \infty$. In this last case, $p_{0}$ and $T_{0}$ clearly become $p_{r}$ and $\theta$ respectively. In the application of this result to a specific air layer, $\alpha$ is fixed by requiring that the ratio $H / L$, that derives from the profile, is equal to its real value in the air layer. Then, $T_{0}$ is fixed by requiring that $H$ (or $L$ ) is equal to its real value as well. Using this method VG obtained a profile for the troposphere that fitted remarkably well the U.S. Standard Atmosphere data. The same method has been applied to the Earth's mesosphere (Pacheco and Sañudo, 2005).

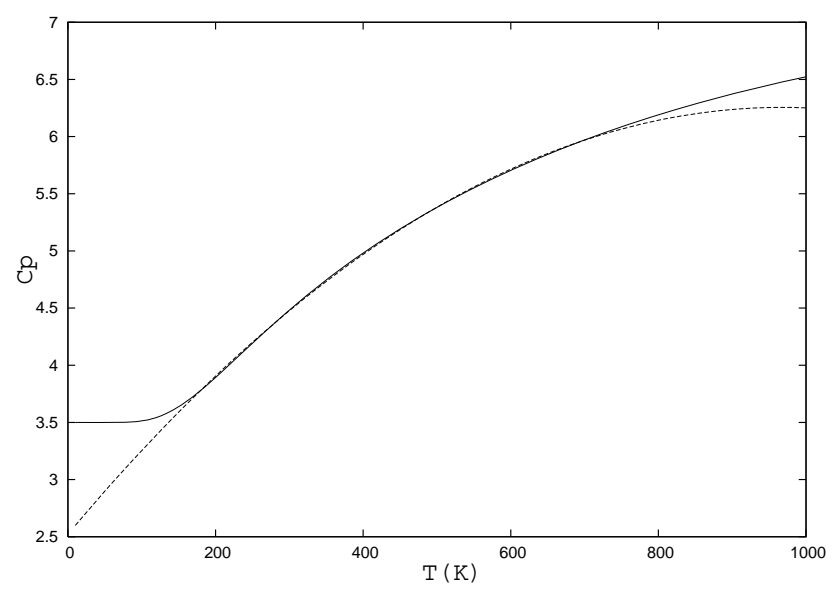

Fig. 1. Specific heat at constant pressure $\left(C_{p}\right)$ in units of $R^{*}$ as a function of temperature (T), of the $\mathrm{CO}_{2}$ molecule (continuous line) and its polynomial fit (dashed line). For details see Sect. 4.

\section{Specific heat of $\mathrm{CO}_{2}$}

The molecule of $\mathrm{CO}_{2}$ is linear and consists of a carbon atom that is doubly bonded to two oxygen atoms. Its molecular mass and corresponding specific gas constant are

$M_{\mathrm{CO}_{2}}=44.01 \mathrm{~g} \mathrm{~mol}^{-1} ; \quad R=M_{\mathrm{CO}_{2}} \times R^{*}=188.92 \mathrm{~J} \mathrm{~kg}^{-1} \mathrm{~K}^{-1}$.

The various contributions to the specific heat, at constant pressure, of a gas constituted by molecules of this type are expressed in the form:

$C_{p}=\frac{7}{2} R+\sum_{T_{\nu}} R\left(\frac{T_{\nu}}{2 T}\right)^{2} \sinh ^{-2}\left(\frac{T_{\nu}}{2 T}\right)$.

The first term in this sum corresponds to translation and to rotational modes while the second term is due to vibrational modes. The sum in the second term extends to the various characteristic temperatures, $T_{v}$, of the vibrational modes. For $\mathrm{CO}_{2}$, these values are (Callen, 1980)

$T_{\nu}(K)=960,960,2000,3380$.

For practical uses, it is convenient to express Eq. (15) by a simple algebraic approximation. Thus, it is standard to express the specific heat by a series of powers in $T$, with coefficients empirically adjusted. Three terms, namely

$C_{p}=A+B T+C T^{2}$,

are enough to describe the specific heat with an excellent accuracy in the range we are interested in. This is clearly shown in Fig. 1, where we have plotted $C_{p}$ vs. $T$ for $\mathrm{CO}_{2}$. The continuous line corresponds to the "exact" values which have been obtained by plotting Eq. (15) with the insertion of the four characteristic temperatures of vibration as given in 


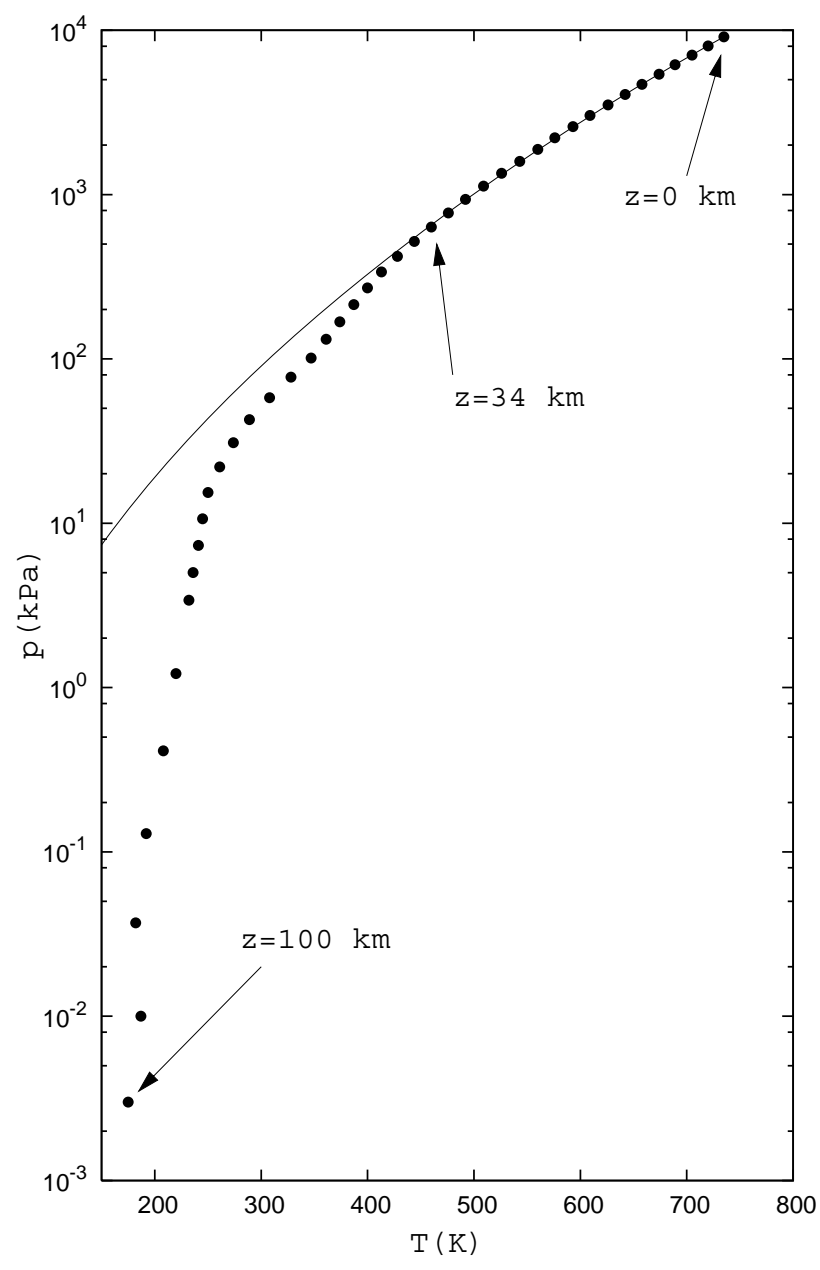

Fig. 2. Pressure-temperature profile of the atmosphere of Venus. The data (black dots) have been taken from Table 1, and the fitting line is an adiabatic as given by Eq. (19).

Eq. (16). The dotted line is the polynomial approximation (17), the coefficients being

$A=2.5223, \quad B=0.7710110^{-2}, \quad C=-0.398110^{-5}$.

The units of $A, B$ and $C$ are $R^{*}, R^{*} / K$ and $R^{*} / K^{2}$ respectively. From Fig. 1 it is apparent, first, that $C_{p}$ has a significant change in the range of temperatures of the Venus atmosphere, from $735 \mathrm{~K}$ at $z=0$ to $175 \mathrm{~K}$ at $z=100 \mathrm{~km}$. Even if we restrict our analysis to the lowest layer, i.e., from $z=0$ to $z=40 \mathrm{~km}$, the interval of temperature is between $735 \mathrm{~K}$ and $385 \mathrm{~K}$. In Fig. 1 one first observes that the change in $C_{p}$ in this interval of temperature is of the order of $50 \%$, and secondly, that this polynomial fit is excellent. Thus, we will use Eq. (17) as input to generalize the VG method and apply it to the atmosphere of Venus.

\section{Potential temperature for a temperature-dependent specific heat}

The generalization of the VG method can start from Eq. (4) because it is applicable to any ideal gas. In this section however, Eq. (5) for the adiabatic transformations, adopts the form

$\int_{\theta}^{T} C_{p}\left(T^{\prime}\right) \frac{\delta T^{\prime}}{T^{\prime}} \equiv \int_{\theta}^{T}\left(A+B T^{\prime}+C T^{\prime 2}\right) \frac{\delta T^{\prime}}{T^{\prime}}=R^{*} \int_{p_{r}}^{p} \frac{\delta p^{\prime}}{p^{\prime}}$.

$p_{r}$ being the pressure level of reference and $\theta$ the potential temperature characteristic of this adiabatic trajectory. We find

$$
\frac{p}{p_{r}}=\left(\frac{T}{\theta}\right)^{A / R^{*}} \exp \left[\frac{B(T-\theta)}{R^{*}}\right] \exp \left[\frac{C\left(T^{2}-\theta^{2}\right)}{2 R^{*}}\right] .
$$

In Fig. 2, we have adjusted the data of the low atmosphere of Venus by means of this formula for an adiabatic. There is a generalized consensus supporting this behavior as can be read in ref. Seiff et al. (1980). It shows an excellent agreement along the lowest $30 \mathrm{~km}$ and a clear departure for upper heights.

In the present case, the physical meaning of the potential temperature is the same as that of Eq. (7), i.e. the temperature that the gas would take if we compressed or expanded it adiabatically to a pressure $p_{r}$. The price to pay now is that given the coordinates $(p, T)$, the value of $\theta$ has to be computed numerically by iterations. Of course, in the case $B=C=0$, Eq. (7) and Eq. (19) are the same equation.

The differential expression for the specific entropy of ideal gases was written in Eq. (8) and now reads

$\delta s=C_{p}(T) \frac{\delta T}{T}-R^{*} \frac{\delta p}{p}$.

For practical purposes, let us define a new magnitude $\tau$, with physical dimensions of temperature, in the following way:

$C_{p}(T) \frac{\delta T}{T}=C_{p}^{0} \frac{\delta \tau}{\tau}$,

with

$C_{p}^{0}=C_{p}\left(T_{0}\right) ; \tau\left(T_{0}\right)=T_{0}$.

In fact, this new variable $\tau$, that reduces to the standard temperature as soon as $C_{p}$ is constant, allows one to treat this problem in exactly the same way as in the case of the ideal perfect gas. 
After specifying $C_{p}(T)$ in a form like Eq. (17), and by the integration of Eq. (21) we obtain

$$
\begin{aligned}
C_{p}^{0} \int_{T_{0}}^{\tau} \frac{\delta \tau}{\tau} & =\int_{T_{0}}^{T}\left(A+B T+C T^{2}\right) \frac{\delta T}{T} \\
= & A\left[\ln \left(\frac{T}{T_{0}}\right)+\frac{B}{A}\left(T-T_{0}\right)\right. \\
& \left.+\frac{C}{2 A}\left(T^{2}-T_{0}^{2}\right)\right],
\end{aligned}
$$

and the result is

$\ln \left(\frac{\tau}{T_{0}}\right)=\frac{A}{C_{p}^{0}} \ln \left\{\frac{T}{T_{0}} \exp \left[\frac{B}{A}\left(T-T_{0}\right)+\frac{C}{2 A}\left(T^{2}-T_{0}^{2}\right)\right]\right\}$.

This is the relation between $\tau$ and $T$. This formula fulfils the two agreed upon conditions: $\tau\left(T_{0}\right)=T_{0}$ and $\tau(T)=T$ for $B=C=0$

Using Eq. (21) in Eq. (20)

$\delta s=C_{p}^{0}\left(\frac{\delta \tau}{\tau}-\frac{R^{*}}{C_{p}^{0}} \frac{\delta p}{p}\right)$,

and in a similar way to what was done to arrive to Eq. (10), we obtain

$s=C_{p}^{0} \ln \tilde{\tau}+$ constant,

with

$\tilde{\tau}=\tau\left(\frac{p}{p_{r}}\right)^{-\kappa^{0}}$

and

$\kappa^{0}=\frac{R^{*}}{C_{p}^{0}}$

It is now clear from this result that in an isentropic process, $\tilde{\tau}$ is also conserved. The use of Eq. (26) will permit us to extend the VG method quite easily, at least from a conceptual point of view.

\section{Generalization of the VG method}

The formal analogy between these new magnitudes and those appearing in Sect. $2, \tilde{\tau} \leftrightarrow \theta, \kappa^{0} \leftrightarrow \kappa$, permits us to extend the VG method reviewed in Sect. 3 in a quite natural way. The entropy, the enthalpy and the $\mathrm{L}$ function of an atmospheric column per unit section are:

$S=\frac{C_{p}^{0}}{g} \int_{p_{2}}^{p_{1}} \ln \tilde{\tau} d p$

$H=\frac{1}{g} \int_{p_{2}}^{p_{1}} h d p=\frac{1}{g} \int_{p_{2}}^{p_{1}} C_{p}(T) T d p$

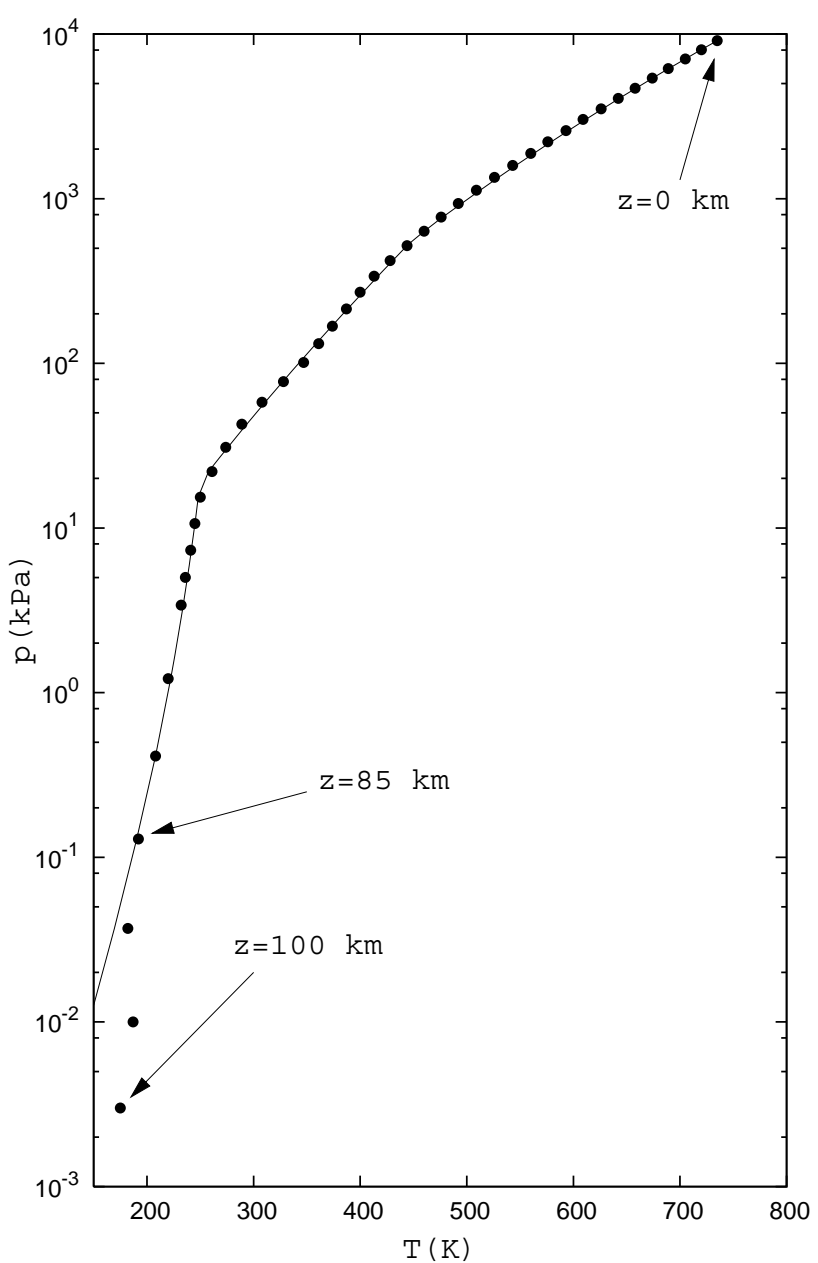

Fig. 3. Pressure-temperature profile of the atmosphere of Venus. The data (black dots) come from Table 1 and the theoretical results (continuous line) have been obtained using the extended VG method.

$L=\frac{C_{p}^{0}}{g} \int_{p_{2}}^{p_{1}} \tilde{\tau} d p$

The condition of maximum $S$, with $H$ and $L$ constants, is expressed as the extreme of the functional $\Psi$ under the variation of the profile $T(p)$, in the following way

$\delta \Psi=\int_{p_{2}}^{p_{1}} \frac{C_{p}^{0}}{g} \int_{p_{2}}^{p_{1}} \delta[\ln \tilde{\tau}+\mu \tilde{\tau}] d p+\lambda \frac{1}{g} \int_{p_{2}}^{p_{1}} \delta[h] d p=0$.

To go into the details of this variational problem, remember that

$\tau=\tau(T) ; h=h(T) ; \quad \tilde{\tau}=\tilde{\tau}(\tau, p)$, 
Then, the condition $\delta \Psi=0$ ( $p$ fixed) implies that

$$
\left(\frac{1}{\tilde{\tau}}+\mu\right) \frac{\delta \tilde{\tau}}{\delta T}+\frac{\lambda}{C_{p}^{0}} \frac{\delta h}{\delta T}=0
$$

and taking into account that

$T C_{p}^{0} \frac{\delta \ln \tilde{\tau}}{\delta T}=\frac{\delta h}{\delta T}$,

one gets

$$
\left(\frac{1}{T}+\mu \frac{\tilde{\tau}}{T}\right)+\lambda=0
$$

Finally, the result of the extreme can be written as

$$
T=T_{0} \frac{1+\alpha}{1+\alpha \tilde{\tau} / T}
$$

with

$\alpha=\frac{\mu}{\lambda} \quad ; \quad T_{0}=\frac{1}{\lambda}$,

being formally identical to the VG result. Nevertheless, it does not provide an explicit expression of $T$ as a function of $p$. This is due to the fact that $\tilde{\tau}$ depends on $\tau$ and through it on $T$, so that $\tilde{\tau} / T$ is now a function of $p$ and $T$. Consequently, the Eq. (37) has to be treated numerically. In fact, by using the method of Lagrange multipliers, the experimental data fix the constraints $H$ and $L$. Then, the parameters $T_{0}$ and $\alpha$ are determined in order to satisfy $H$ and $L$.

\section{Results of the extended VG method}

The performance of the extended VG method, summarized in Eq. (37), when applied to the atmosphere of Venus is shown in Fig. 3. Note that the logarithmic scale used for pressure is more sensitive and hence puts more clearly in evidence the virtues or flaws of the fit. The characteristics of the Venus atmosphere allow us to well distinguish three regions in altitude. In each one of these regions we have applied the extended VG method by maximizing the entropy with both $H$ and $L$ constraints.

In the lowest one, from $z=0$ to $z \simeq 34 \mathrm{~km}$, we found that the value of $\alpha$ is 44 and the value of $T_{0}=735 \mathrm{~K}$. This high value of the unique parameter of the model indicates the clear preponderant role of $L$ with respect to $H$, as the dominant constraint in the maximization of $S$. From this fact we conclude that in this layer the profile is adiabatic. This clearly agrees with the result shown in Fig. 2, obtained by means of Eq. (19).

In the layer from $z \simeq 60 \mathrm{~km}$ to $z \simeq 100 \mathrm{~km}$, the value obtained for $\alpha$ is 0.18 and $T_{0}=245 \mathrm{~K}$. This indicates that here $H$ is the dominant constraint, and in consequence the profile of this layer is basically isothermal.

The method assigns $\alpha=3$ with $T_{0}=444 \mathrm{~K}$ to the intermediate layer, a transition region, from $z \simeq 34 \mathrm{~km}$ to $z \simeq 60 \mathrm{~km}$.

\section{Discussion and conclusions}

Our purpose in this paper was to extend the first-principles VG method to atmospheric layers where the thermal deviation is so high that it is not reasonably possible to consider a constant unique value for the specific heat in all the points of the layer. To implement this extension, we have had to define a new extended potential temperature, $\tilde{\tau}$. A potential temperature is, by definition, a generalized temperature that characterizes adiabatic processes, or in other words, that is conserved in processes where the entropy is constant. The relation between the new $\tilde{\tau}$ and entropy maintains the same standard form as that existing for ideal-perfect gases.

From the surface of Venus up to about $100 \mathrm{~km}$, we have distinguished 3 layers. The extended VG method, for each layer, after fixing $T_{0}$ to the corresponding initial temperature of the layer, deals with a unique parameter $\alpha$ to adjust the constraints. From the surface up to $35 \mathrm{~km}$, the method assigns a high value of $\alpha$. This implies that the $p-T$ profile here is adiabatic. There is no surprise in this result; an adiabatic lapse-rate was detected in the low atmosphere of Venus during the first observations of the Russian Venera probes. This was verified later in other spatial missions (Seiff et al., 1980). In the layer from 35 to $65 \mathrm{~km}$ in height $\alpha$ has an intermediate value that indicates a transition. Finally, from 65 to about $100 \mathrm{~km}$, this method produces a low $\alpha$, which is interpreted as an isothermal layer. This is the first time that the VG method identifies an adiabatic layer where it should; this concordance shows clearly the success of the method itself and of the extension presented in this paper.

Acknowledgements. L. N. Epele, H. Fanchiotti and C. A. García Canal acknowledge CONICET and ANPCyT of Argentina for financial support. A. F. Pacheco and J. Sañudo thank the Spanish DGICYT for financial support (Project FIS 2005-06237).

Edited by: T. Chang

Reviewed by: two anonymous referees

\section{References}

Bohren, C. F. and Albrecht, B. A.: Atmospheric Thermodynamics, Oxford Univ. Press, 1998.

Callen, H. B.: Thermodynamics, John-Wiley and Sons, p. 323-327, 1980.

Landis, G., Colozza, A., and LaMarre, C.: Atmospheric flight on Venus, NASA-2002-0919, 2002.

Pacheco, A. F. and Sañudo, J.: A maximum entropy profile for the mesosphere, Nuovo Cimento, C28, 29-32, doi:10.1393/ncc/i2005-10021-9, 2005.

Seiff, A., Kirk, D. B., Young, E., et al.: Measurements of Thermal Structure and Thermal Contrasts in the Atmosphere of Venus and Related Dynamical Observations: Results from the Four Pionner Venus Probes, J. Geophys. Res., 85, 7903-7933, 1980.

Staley, D. O.: The adiabatic lapse rate in the Venus atmosphere, J. Atmos. Sci., 27, 219-223, 1970. 
L. N. Epele et al.: Venus atmosphere profile from a maximum entropy principle

Tsonis, A. A.: An Introduction to Atmospheric Thermodynamics, Cambridge Univ. Press, 2002.
Verkley, W. T. M. and Gerkema, T.: On Maximum Entropy Profiles, J. Atmos. Sci., 61, 931-936, 2004. 